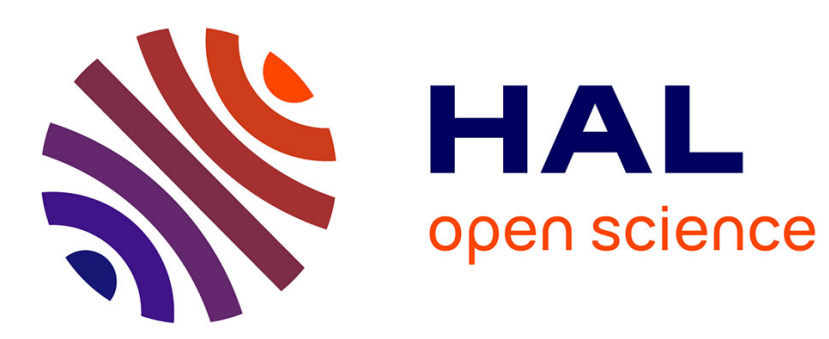

\title{
L'intervention-recherche en milieu de travail enseignant comme moyen de formation
}

\author{
Christine Felix, Frédéric Saujat
}

\section{To cite this version:}

Christine Felix, Frédéric Saujat. L'intervention-recherche en milieu de travail enseignant comme moyen de formation. Raisons Educatives, 2015, 19, pp.201-218. hal-01755763

\section{HAL Id: hal-01755763 \\ https://hal.science/hal-01755763}

Submitted on 30 Mar 2018

HAL is a multi-disciplinary open access archive for the deposit and dissemination of scientific research documents, whether they are published or not. The documents may come from teaching and research institutions in France or abroad, or from public or private research centers.
L'archive ouverte pluridisciplinaire HAL, est destinée au dépôt et à la diffusion de documents scientifiques de niveau recherche, publiés ou non, émanant des établissements d'enseignement et de recherche français ou étrangers, des laboratoires publics ou privés. 


\title{
L'intervention-recherche en milieu de travail enseignant comme moyen de formation
}

\author{
Marie-Christine Felix \& Frédéric Saujat \\ Aix-Marseille Université
}

Comme le rappellent Leblanc, Ria, Dieumegard, Serres et Durand (2008), depuis une dizaine d'années des échanges et des collaborations ont vu le jour entre les sciences de l'éducation et les sciences du travail. Au-delà de leurs différences théoriques, les approches fondant ces échanges sont sous-tendues par le postulat qu'une condition d'efficacité de la formation est de prendre en compte le travail réel des professionnels de l'éducation (Saujat, 2007). En posant les termes d'une articulation renouvelée entre l'exercice du métier et les conditions susceptibles de préparer à cet exercice et de le soutenir, elles assignent au travail réel tantôt le statut d'objet à comprendre (afin de le transformer), tantôt, à travers son analyse, celui d'instrument de formation. Dans ce chapitre, nous nous intéressons à ces deux modalités des relations entre analyse du travail et formation, en nous appuyant sur une intervention conduite en milieu de travail enseignant.

\section{Une intervention à visée de formation : dispositif et présupposés théoriques et méthodologiques}

Les matériaux sur lesquels nous nous appuyons sont issus d'une intervention en milieu de travail, mise en œuvre pour répondre à une demande de formation émanant d'un collège situé en Zone d'éducation prioritaire ${ }^{1}$ (Felix \& Saujat, 2007). La mise en œuvre d'un tel processus s'inscrit pour nous dans la tradition de l'intervention ergonomique (Daniellou, 1996, 2007). Comprendre le travail pour le transformer, à la demande des intéressés et avec leur concours, est aujourd'hui une règle de métier admise dans le champ de «l'ergonomie de l'activité » qui considère que le travail ne peut être seulement regardé ni du point de vue de la prescription ni du point de vue de ses résultats.

La demande de formation émane du chef d'établissement, en poste depuis un an, et elle est fondée sur le constat suivant : «On n'est pas bons ! Les performances scolaires des élèves se

\footnotetext{
${ }^{1}$ Zone dans laquelle sont implantés des établissements scolaires dotés de moyens supplémentaires et d'une certaine autonomie pour faire face à des difficultés d'ordre scolaire et social.
} 
dégradent entre la $6^{\mathrm{e}}$ et la $3^{\mathrm{e}}$. Pourtant nous avons une équipe de profs motivés et une vie scolaire d'enfer. Comment assurer la continuité des apprentissages des élèves face à l’hétérogénéité de ce public ?».

Lors de notre première rencontre avec cinq professeurs qui représentent les enseignants du collège et qui constitueront le collectif restreint (voir fig. 1), la question de l'hétérogénéité est reprise. Ils disent se trouver démunis pour «gérer » cette hétérogénéité et faire en sorte que tous les élèves entrent dans les activités proposées. Au-delà de ce consensus, ils disent aussi ne pas se trouver tous dans la même situation, selon les disciplines enseignées, les moments de la journée ou le niveau des classes. L’expérience professionnelle est également évoquée, surtout par les débutants qui pensent rencontrer des difficultés d'une autre nature que celles des professeurs plus chevronnés : prendre la classe en main, se faire respecter, mettre les élèves au travail, autant de problèmes de discipline que n'auraient pas ces derniers.

À ce stade, comme insistait Wisner (1995), la demande permet de pointer sur la difficulté, mais la variabilité des situations de travail fait que la résolution du problème ne saurait être immédiate tant ce dernier, loin d'être donné, reste à construire avec ceux qui le posent. Pour ce faire nous avons proposé aux membres du collectif restreint un cadre dialogique, instrumenté par l'image vidéo (Faïta, 2007), propre à constituer leur travail en objet de pensée et d'interlocution, par l'entremise d'un processus de co-analyse et de co-construction de «faits » (Daniellou, 1995) qui soient discutables par le collectif élargi comprenant l'ensemble des professeurs de l'établissement. Nous avons également établi un contrat, fixant la durée de l'intervention, les modalités de la collaboration et d'observation dans les classes, l'usage des images vidéo dans les différentes phases (autoconfrontations simples - ACS - et croisées ACC), la fréquence des rendez-vous avec les membres du collectif restreint, ainsi que l'organisation des phases de restitution du produit des autoconfrontations auprès du collectif élargi, à raison d'une journée par trimestre banalisée par le chef d'établissement pour libérer les professeurs de leurs cours.

Le processus de co-analyse ainsi initié se déroule selon plusieurs cycles et plusieurs contextes qui jalonnent les étapes de l'intervention. La figure 1 présente un premier cycle dans lequel la constitution du groupe restreint est de première importance puisqu'il lui incombe de définir, avec les intervenants, l'objet de co-analyse qui est choisi dans les séquences filmées. Les difficultés à faire classe avec ce public hétérogène est $l^{\prime}$ Objet $\mathrm{n}^{\circ} 1$ retenu pour être soumis à l'analyse.

Figure 1 : Déroulement de l'intervention - Cycle 1 


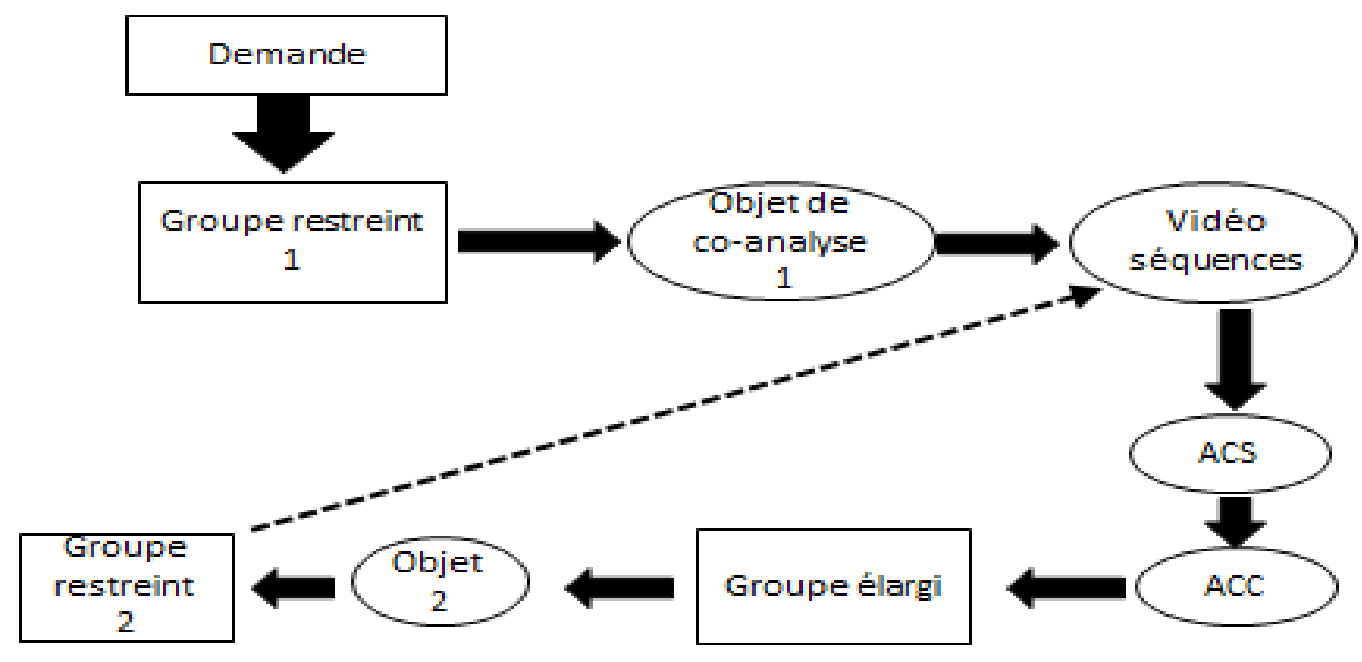

L'étape suivante consiste à filmer, pour chacun des professeurs, cette "entrée en classe », puis à confronter chacun d'eux à sa propre activité au cours d'une ACS. Celle-ci implique un sujet d'abord confronté à lui-même à travers les actions qu'il se voit réaliser. Chacun s'engage donc, au cours de cette première phase, dans un dialogue avec lui-même dans le cadre duquel il justifie ou évalue ses propres actes, s'en étonne éventuellement et en construit une vision nouvelle, avant d'être confronté, dans une deuxième phase d'ACC, à l'évaluation par un pair de ses actes de travail aussi bien que de ses commentaires, en présence de l'intervenant.

L'originalité de l'autoconfrontation, en tant que méthode de sollicitation de l'expérience et des savoirs en actes, réside dans la libération des façons de signifier offertes aux sujets. En effet, les énoncés produits en autoconfrontation s'inscrivent dans un réseau de rapports dialogiques (Bakhtine, 1984) qui se déploient à plusieurs niveaux (Faïta \& Vieira, 2003). Adressé $\mathrm{au}(\mathrm{x})$ destinataire(s) immédiat(s), dont le locuteur attend compréhension et réponse, chaque énoncé l'est aussi au locuteur lui-même, à travers ce que Bakhtine (1970) appelle le « microdialogue » (p. 362), ainsi qu'à un «sur-destinataire », troisième participant invisible : en autoconfrontation il s'agit du métier, comme instance «qui se situe au-dessus de tous les participants du dialogue »(Bakhtine, 1984, p. 337). Dans ce cadre, les actes de travail saisis par le film ne sont pas seulement des prétextes à commentaires ou explications dirigés vers autrui : ils prennent sens d'une façon souvent insoupçonnée jusqu'alors par leurs propres auteurs. La réappropriation dont ils sont l'objet conduit les sujets à produire des discours qui 
ne sont jamais seulement la contrepartie verbale ou l'explicitation des actes visualisés. Un sujet éprouve ainsi presque toujours le besoin, en sollicitant l'arrêt du défilement de l'image, de faire place à ce « concentré d'histoire qu'est chaque situation d'activité » (Schwartz, 2000, p. 735) : ce qui s'est déjà passé, déjà fait et qui justifie ce que l'on voit, ce qui doit advenir ensuite et impose aux actes telles caractéristiques, ce qui pourrait être fait autrement, par d'autres ou par le sujet lui-même...

Une fois ces autoconfrontations réalisées, les protagonistes font le choix de séquences vidéo significatives du point de vue de la demande, celle adressée aux intervenants, et discutables du point de vue du collectif. Le montage ainsi réalisé constitue l'artefact méthodologique (Faïta, 2007) instrumentant le débat et la réflexion au sein du collectif des enseignants de l'établissement. À l'issue de la journée trimestrielle, ce dernier propose, sur la base des débats et des réponses et ressources à construire, de travailler sur un nouvel objet (Objet $\mathrm{n}^{\circ} 2$ dans la figure 1) à partir duquel d'autres professeurs deviennent membres d'un autre groupe restreint (Groupe restreint $\mathrm{n}^{\circ} 2$ dans la figure 1). Ce cycle trimestriel permet d'enrôler à chaque fois de nouveaux protagonistes et d'élargir le collectif initialement composé des cinq professeurs.

La figure 2 présente un second cycle coordonné au premier. Elle montre que la série des autoconfrontations à laquelle participe chaque membre du groupe restreint est entrecoupée par des retours en classe. Chaque participant connait en quelque sorte une double alternance répétée selon un double mouvement, externe et interne :

- une alternance « externe » relative au dispositif mis en place, entre expériences de classe et expériences liées aux processus d'interaction formative (Bronckart, 2001) qui se déploient à la faveur du processus d'intervention ;

- une alternance «interne » liée à l'activité des protagonistes eux-mêmes, entre ce que l'enseignant fait dans son milieu ordinaire de travail - la classe -, ce qu'il dit dans le milieu extra-ordinaire de l'autoconfrontation à ce qu'il fait en classe, et pour finir ce qu'il fait de ce qu'il a dit lors de son retour en classe.

Dans cette alternance répétée, ce qui a été fait de ce qui a été dit est donc confronté à une double mise à l'épreuve : celle du travail réel en classe et celle de son analyse en retour.

Figure 2 : Alternance autoconfrontations / travail en classe - Cycle 2 


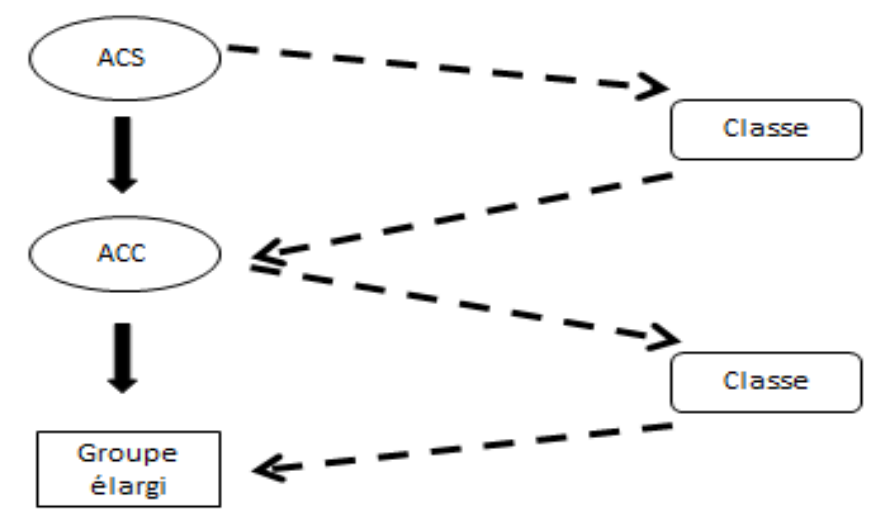

Au-delà d'interactions répétées entre les protagonistes, au cours desquelles chacun s'engage dans les dialogues multi-adressés évoqués plus haut (à lui-même, à l'intervenant, à un [des] pair[s] et au métier), ce sont les aller-retours résultant de la mise en œuvre de ces deux cycles qui permettent la relance de l'activité individuelle et collective. En effet, la reprise de l'expérience vécue que nous cherchons à organiser consiste en un développement de cette dernière, par le sens qu'elle prend dans la pluralité de contextes d'analyse et d'action qu'elle traverse et dans lesquels les professionnels trouvent matière à expérimenter ce dont ils sont capables (Canguilhem, 1930). Nous suivons ainsi Clot (2000), pour qui l'analyse du travail est un moyen de formation à la condition de devenir un instrument de transformation de l'expérience. Il s'agit alors d'une formation elle-même transformée en travail. Ce «travail sur le travail » a pour effet de produire un changement de statut du vécu, à la source d'un développement du pouvoir d'agir : «d'objet d'analyse, le vécu doit devenir moyen pour vivre d'autres vies »(Clot, 2000, p. 154). Nous rejoignons également Jobert (2000) lorsqu'il souligne que dans la métaphore de la formation comme développement, dont l'apparition est liée selon lui à l'importance des questions pratiques et théoriques laissées sans réponses par les représentations précédentes de l'apprentissage et du développement en formation d'adultes, et particulièrement en formation professionnelle, «travail et formation entretiennent [...] des relations complexes d'engendrement réciproque » (p. 23). Il ajoute que cette métaphore « ouvre au formateur de vastes possibilités d'intervention visant l'exploration de l'expérience à des fins de développement de la personne et de transformation des situations de travail et de vie » (p. 25). 


\section{L'autoconfrontation et ses effets}

Jeune enseignante d'histoire et géographie, néo-titulaire, Guillemette dit rencontrer d'importants problèmes d'autorité, en particulier avec une classe de $6^{\mathrm{e}}$. L'ACS, dont nous présentons infra un extrait, se déroule le lendemain du filmage d'une leçon de géographie qui porte sur les «zones de peuplement en France ». On voit sur la vidéo que les élèves entrent en classe de manière désordonnée, bousculent chaises et bureaux et s'interpellent bruyamment. Guillemette indique au cours de l'ACS qu'elle décide alors de les faire sortir de la classe, « d'attendre qu'ils se mettent correctement en rang dans le couloir, devant la porte, et qu'ils rentrent en silence de manière à pouvoir commencer à travailler dans de bonnes conditions ». Les élèves entrent à nouveau, se tiennent debout, à côté de leur chaise et attendent que la professeure leur donne l'autorisation de s'asseoir. Elle «attend le silence absolu » et procède à des rappels de règles : les places assignées aux élèves, la prise de parole, des consignes de bonne tenue du cahier d'histoire-géographie. Elle enchaine sur le démarrage du cours alors que les élèves ne sont ni assis ni silencieux.

Confrontée aux «traces » de son activité et sollicitée par le chercheur, Guillemette (G) tente de donner du sens à l'activité qu'elle déploie en classe, avec ses élèves. Dès les premières images, elle fait le constat, non sans souffrance, que le «bras de fer » qu'elle est en train d'établir malgré elle, avec les élèves, la conduit vers une impasse, retardant plus encore le moment d'asseoir son autorité et d'organiser le milieu dans lequel les élèves vont pouvoir étudier la notion en jeu.

\section{Autoconfrontations simple et croisée}

Le premier acte consécutif à la réalisation de l'autoconfrontation consiste à se saisir du texte co-produit comme d'un objet de nature dialogique (Bakhtine, 1977, 1984 ; Faïta, 2007). Comme le note François (1996), «parler de "dialogisme" est donc ici synonyme $\mathrm{d}$ "interprétation" [...] au sens de ce qui rend capable de faire entrer un texte ou un objet signifiant en général dans des ensembles où ils font sens » (p. 13). Nous suivons également cet auteur qui précise que «texte» ne signifie pas ici texte écrit, mais « un énoncé ou un ensemble d'énoncés [...] dans sa triple relation aux autres textes, à notre compréhension responsive, aux effets de mise en mots dont il est capable » (p. 14). Ces effets se produisent dans un espace de jeu où il y a de l'ouvert, de l'inattendu, espace justiciable d'une linguistique de l'événement en quête de la diversité des façons de signifier qui se manifeste dans la circulation du sens (François, 1993). Nous sommes donc attentifs à ces 
manifestations ${ }^{2}$, en portant un intérêt particulier à celles qui renvoient à l'affectation du sujet lorsqu'il se mesure aux traces de son activité (Bournel Bosson, 2011) ${ }^{3}$.

G. : c'est un gros problème que j'ai avec cette classe... il n'y en a pas que un ou deux il y en a sept... ou huit et c'est récurrent et ça me... mais j'ai réussi enfin à prendre quelques élèves à part et à la fin à mettre des mots dans le carnet parce que je me dis j'ai fini par me rendre compte que chez eux mon autorité n'était vraiment pas respectée parce qu'ils se permettaient de me répondre ... oui c'est là que j'ai un problème...

Intervenant (I.): Euh et juste encore une question tu fais malgré tout le choix de commencer à leur donner les consignes

G. : Là je me rends compte que c'est une erreur c'est une erreur

I. : Euh c'était pas délibéré au moment où tu l'as fait c'était pas...

G. : Euh non non j'avais peut être pensé depuis l'autre bout de la classe qu'il n'y avait pas tant de bruit que ça et puis surtout ça faisait dix minutes qu'on était rentré et qu'il faut bien que je commence à un moment donné mais là non c'est pas c'est pas possible...

(Le visionnage reprend)

G. : Là enfin on a à peu près quelque chose qui redevient normal et je me rends compte que j'ai pas... à les laisser debout comme ça... maintenant je comprends mieux pourquoi il y avait tant de bruit de chaises... quand ils sont debout ils font un pas sur l'autre un pas sur l'autre donc j'ai peut-être intérêt à leur dire rapidement de s'asseoir donc de faire en sorte d'avoir un silence peu importe qu'il soit absolu ou pas... mais assez vite pour que vite ils s'assoient que vite on démarre parce qu'en fait je perds du temps à obtenir quelque chose que j'arriverai pas à obtenir et du coup ça contribue à...

La préoccupation de Guillemette est qu'elle ne parvient pas à faire preuve d'autorité avec cette classe, ce dont elle témoigne en début d'entretien, jusqu'à ce que le chercheur remarque qu'elle commence à donner des consignes alors que le retour au calme qu'elle réclamait ne s'est pas fait. Le dialogue ainsi engagé ré-oriente l'activité de Guillemette depuis le « problème » qu'elle avait vers la prise en compte du «temps » qu'elle perd à vouloir le régler. Elle «comprend mieux » à ce moment-là les raisons qui occasionnent le bruit et entrevoit le moyen d'obtenir non pas le silence absolu mais la possibilité de démarrer rapidement.

$\mathrm{G}$ : Par contre je me rends compte que sur une phrase que je voulais rapide que je passe un temps un peu trop long... démesurément trop long et ça parce que comme mon souci... on a un programme et on fait des choses trop longues au début et après on s'attache à raccourcir de plus en plus et à adapter de plus en plus aux élèves et là j'aurais

\footnotetext{
${ }^{2}$ «On ne peut parler ici de signifiants et de signifiés, mais plutôt de manifestants et de manifestés, parce que, selon la façon dont il est interprété, le même manifestant ne renverra pas au même manifesté, le même discours, par exemple, sera manifestation de l'objet dont on parle, de soi-même, de l'interlocuteur. D'autre part, parler de manifestation veut dire qu'il y a là une interprétation, non des signes qui seraient a priori destinés à signifier de telle ou telle façon. Enfin un manifesté, par exemple le monde dans lequel l'objet dont on parle prend forme, pourra être à son tour un "manifestant" de soi en tant que locuteur » (François, 1989, p. 45).

${ }^{3}$ Les marques de cette auto-affectation sont en caractères gras.
} 
dû... je sais pas quoi... on aurait dû passer à autre chose depuis bien longtemps...simplement parler avant leur faire réfléchir écrire reparler après...

I.: C'est pas un moyen quand même de les amener à s'approprier les concepts qui sont derrière... je trouve intéressant d'expliciter un certain nombre de termes qui peuvent poser problème...

G. : Ouais mais je me demande si je pourrais pas faire la même chose en plus rapide quand même parce que je vois tout le temps passé sur une seule phrase et puis toutes les notions à faire tous les exercices à faire et les autres notions à passer... ça me parait un peu long quoi...

Dans la suite du visionnage, Guillemette découvre que «ça travaille » malgré un «mauvais départ », un bruit résiduel, la caméra... Elle se voit elle aussi travailler et se rend compte du décalage entre ce qu'elle voulait faire rapidement et le temps trop long mis à le réaliser. L'intervenant remarque alors que passer du temps à présenter des concepts ou des notions n'est peut-être pas inutile pour les élèves, et elle enchaine en énonçant qu'elle perçoit la possibilité de «faire la même chose, mais en plus rapide ».

Cet extrait d'ACS illustre des effets récurrents au fil de nos interventions. Il donne à voir l'évolution suivante : découvrir la singularité de ce qu'on fait, se découvrir comme sujet agissant faisant des choix et prenant des décisions qui n'étaient pas les seuls possibles, construire ses propres repères en fonction desquels ce qu'on fait peut être évalué et mis en perspective... Cette évolution concerne des objets de pensée et d'interlocution qui se déplacent : perdre du temps à exiger et attendre le silence complet apparaît brusquement à Guillemette comme inutile voire contreproductif du point de vue de la reconnaissance par les élèves de son autorité. Elle passe ainsi de la manifestation d'une préoccupation inhibitrice («comment asseoir son autorité ») à l'analyse de son «occupation » qu'elle peut envisager dans une perspective dynamique, c'est-à-dire du point de vue des rapports mouvants entre ce qu'elle se voit faire, ce qu'elle voudrait faire et ce qu'elle pourrait faire.

Cet extrait montre aussi que cette évolution est liée aux interventions de l'intervenant qui s'efforce de confronter systématiquement Guillemette à ses dilemmes ou conflits de critères. Cela lui permet de se déprendre de sa position de commentatrice, qui dit ce qu'elle fait, pour se positionner comme une interlocutrice capable de «ré-agir », en prise avec la motricité du dialogue. Et elle le fait en passant de «je me rends compte... » à «je comprends mieux...» pour enchaîner sur « je me demande si... ».

L'extrait suivant met en présence Guillemette, Frédérique (F) - qui est professeure de lettres, en poste depuis quatre ans dans le collège au moment de l'intervention et qui a bénéficié elle aussi d'une ACS -, et l'intervenant, dans le cadre d'une ACC sur le film de Guillemette à propos de la même séquence. 
G. : alors moi ce que je trouve intéressant dans mon cours c'est que c'est l'archétype du cours qui commence mal parce que c'est trop... cela demande une attention à ces gamins qui ne peuvent pas donner tout de suite... il y a eu trop de moments de flottements au début, on n'est pas encore installé et c'est vrai que... projeter un document $[\ldots]$ où il y a quelque chose à regarder un support quelque chose qui les accroche... mais là ce que je trouve intéressant c'est cette mise en activité si longue car il faut rappeler le cours précédent, parce qu'il faut faire attention à vérifier le travail [...] là ça va mieux mais la première fois que j'ai vu ça j'étais horrifiée par...

Guillemette s'adresse à sa collègue Frédérique et ses propos évoquent à la fois le film de la classe et l'ACS où elle déplorait cette mise en activité trop longue, qu'elle qualifie maintenant « d'intéressante ». Elle reprend l'ouverture envisagée au cours de l'ACS, mais cette fois sur un mode qui n'est plus interrogatif [ «je me demande si je pourrais pas faire la même chose en plus rapide »], mais assertif [« tout ça je pourrais le dire plus vite et d'une autre manière »].

F : et ça tu t'en es vraiment rendu compte en...

$\mathrm{G}$ : en regardant ouais ouais à quel point c'était long à quel point il y avait du brouhaha au début du cours et c'est vrai que cela m'a vraiment aidée...

[Le film reprend]

G : quoi ? Je dicte encore !

$\mathrm{F}$ : avance avance ne te fais pas souffrir...

$\mathrm{G}$ : en fait je comprends pourquoi ils sont silencieux... en fait je l'avais pas vu comme ça la première fois... en fait je ne leur demande rien alors ils écrivent ils écrivent... sans penser...

[Le film reprend]

G. : et du coup je me rends compte après coup qu'ils étaient bien indulgents de continuer à m'écouter... relativement...

Le contexte de l'ACC permet à cette jeune professeure de revivre une expérience d'une autre manière, dans le cadre d'un nouvel adressage : «je ne l'avais pas vu comme ça la première fois...». Elle découvre un événement passé inaperçu jusqu'à présent, «quoi je dicte encore ? », source d'un nouvel étonnement qui l'amène à établir une relation entre sa propre activité - dicter - et celle des élèves - écrire en silence. Mais la conséquence de ce nouvel étonnement, c'est qu'elle considère avec d'autres yeux des élèves qui ne la respectaient pas (dans l'ACS) et qui écrivent sans penser (dans l'ACC). Et ce nouveau pas en avant la place face à d'autres contradictions : elle obtient le silence sous la dictée, mais ce faisant les élèves «ne pensent pas ». En progressant dans la confrontation avec soi-même, par l'entremise du pair ou de l'intervenant, Guillemette interroge ses choix, ses partis pris à partir des compromis opératoires qu'elle tente de réaliser. Mais ces compromis ne se limitent pas aux seules dimensions opératoires avec lesquelles elle essaie de composer. Ils sont liés à la fois aux 
prescriptions (laisser une trace écrite du cours) et au sens des activités respectives de Guillemette et de Frédérique.

F. : alors là moi c'est un truc j'ai tellement la phobie du cours magistral que... parce que moi les cours d'histoire j'ai jamais aimé parce que j'ai toujours eu des cours comme ça...

G. : c'est clair

F. : et j'avais un mal fou à essayer de rester concentrée une heure... c'est une phobie que j'ai...alors moi j'ai le problème inverse, tu vas voir tout à l'heure c'est tellement... ouais c'est la fête on dialogue et machin... qu'à la fin... qu'à un moment la trace écrite on la fait au cours suivant... parce que je me suis emballée et qu'il n'y a plus rien et du coup j'ai rien dicté et puis la gestion du temps... moi je merde complètement tu verras la sortie de classe c'est quelque chose... alors après c'est un problème inverse

Ici s'opère une bascule. C'est Frédérique qui prend la parole pour dire qu'elle fait l'inverse de Guillemette et que c'est un problème pour elle, parce qu'elle n'a rien dicté, n'a pas su gérer le temps, etc. «Phobique» du cours magistral, elle choisit de faire un cours vivant, avec pour conséquence l'absence de trace écrite du cours. En fait Frédérique, qui présente aux yeux de Guillemette l'assurance du professeur confirmé et qui n'a pas de problème d'autorité dans ses classes, ne parvient pas mieux qu'elle à trouver des compromis opératoires satisfaisants. Cette activité empêchée autant pour l'une que pour l'autre témoigne d'une difficulté commune : comment composer avec la place de l'écrit dans l'activité des élèves et la construction des connaissances, et l'écrit comme but de la séance (laisser une trace écrite du cours) ? Et plus largement d'un problème professionnel lié à la gestion du temps.

\section{Retour au collectif élargi}

Le retour au collectif élargi se fait sur la base d'une sélection des extraits retenus par les protagonistes du collectif restreint. Le matériau composé d'images de situations de classe et d'autoconfrontations des professeurs relatives à ces situations devient ainsi le support et l'instrument des échanges initiés dans le collectif élargi. Les manières de faire et de penser sont ainsi mises en discussion, adoptées, adaptées ou récusées par ce collectif.

Dans la situation que nous rapportons, le collectif restreint propose des extraits concernant, entre autres professeurs, Guillemette et Frédérique.

Confrontés aux images de leurs collègues, plusieurs professeurs déclarent se trouver dans la même situation que celle rapportée à travers les vidéos. Les difficultés que Guillemette vivait sur un mode personnel se convertissent alors pour elle en un questionnement partagé par un collectif. Les professeurs se rendent compte que, comme leurs collègues du collectif restreint, la cloche sonne avant qu'ils n'aient réussi à faire produire une trace écrite, à noter sur le 
cahier de texte les devoirs à faire, etc. Si bien qu'ils sont amenés à prendre du temps supplémentaire pour terminer ce travail, ce qui raccourcit d'autant la durée du cours suivant, et place les élèves dans des dispositions peu favorables, aussi bien pour le cours suivant que pour le prochain cours dans la même discipline. Un nouvel objet de travail émerge alors, désigné au sein du collectif par l'expression : «lâcher la classe », et articulé à l'Objet $\mathrm{n}^{\circ} 1$ «prendre la classe », mais aussi à d'autres objets émergents comme "gestion du temps », «terminer le cours », «trace écrite »... Le collectif se remet au travail sur ces objets dans plusieurs directions qui réactivent des sous-entendus du métier pour les mettre en débat. Ici aussi le rôle de l'intervenant est de susciter la confrontation, mais également d'étayer l'effort du collectif dans son travail d'élaboration. Des extraits de films de classes réalisés dans le primaire (élèves de 6 à 11 ans) sont proposés comme des relances à des interrogations relatives à la correction d'exercices en classe ou à l'organisation du travail en groupe. Les écarts entre ces niveaux d'enseignement conduisant les professeurs à reformuler ou à préciser leur point de vue, ou encore à faire valoir des divergences dans lesquelles il reste à trancher.

Dans les rapports individu / collectif, on a vu comment a évolué Guillemette dans sa capacité à repérer progressivement les impasses et les moyens de les surmonter au cours des autoconfrontations. Avec le retour au collectif, ce développement va connaitre une nouvelle avancée. Pendant cette journée, elle a pris beaucoup de notes, elle est souvent intervenue, et le travail de ce collectif devient pour elle un outil d'appropriation de ressources :

G. : si je comprends bien mettre les élèves en groupes ce n'est pas les mettre deux par deux mais c'est organiser des groupes où chacun a une fonction bien définie... vu comme ça cela permettrait de gérer l'hétérogénéité...

Au même titre, l'autoconfrontation devient un outil au service de nouveaux apprentissages professionnels :

G. : je voudrais vraiment essayer de mettre ça au point dans ma classe et j'aimerais que tu viennes me filmer... j'aimerais voir ce que cela donne...

Ainsi le processus d'intervention, qui crée un rapport inédit dans le milieu ordinaire de travail entre un groupe restreint et un collectif professionnel, permet au premier de relancer l'histoire du second dès lors que les professeurs s'emparent des difficultés et des moyens d'y répondre en commun grâce à l'institution de ce que l'on peut appeler, à la suite de Mayen (1999), une situation potentielle de développement. Cette situation ouvre sur la possibilité de remettre en discussion des choix «arrêtés », de réactualiser des sous-entendus du métier. Ils sont non seulement mis en débat mais débouchent aussi sur la définition d'un nouvel objet, validé cette 
fois par le collectif des enseignants : «comment organiser l'activité des élèves en classe pour gérer l'hétérogénéité ?» (appelé Objet $\mathrm{n}^{\mathrm{O}} 2$ dans la figure 1). À cet égard, nous faisons l'hypothèse que c'est pour mieux contribuer au travail collectif, c'est-à-dire s'expliquer avec sa propre activité, avec celle des autres et les passer au crible du métier, que les professeurs sont de plus en plus nombreux à demander à participer aux autoconfrontations à l'issue du retour au collectif (Groupe restreint 2 dans la figure 1).

Entre les différentes phases de l'intervention (ACS, ACC, retour au collectif), le retour en classe est donc pour Guillemette un lieu où peuvent se réaliser des apprentissages nouveaux. À l'issue de ce premier cycle, elle s'engage notamment dans la mise en place de groupes de besoins différents selon le degré de réussite des élèves à l'épreuve d'un brevet blanc : travail en trois groupes (autonome, guidé par des sources documentaires supplémentaires, et dirigé par elle pour les élèves les plus en difficulté). Cette situation de travail sera filmée et objet d'ACS, ACC, et soumise au collectif lors du deuxième retour au collectif élargi que nous ne présentons pas ici.

En résumé, on peut dire que le travail entrepris avec Guillemette met en évidence la manière dont la traversée d'une pluralité de contextes d'analyse et d'action et la diversification des adressages qui multiplient les contacts sociaux avec elle-même, pour reprendre une expression vygotskienne, contribuent à produire a) des étonnements, des discordances vécues qui font «ré-agir» les protagonistes, b) un déplacement et un recyclage potentiel des préoccupations liés à des déplacements de perspective (Saujat \& Serres, 2015) et c) des cycles récursifs d'apprentissage et de développement professionnels liés à une relance du pouvoir de (re)penser ses occupations, un pouvoir de ré-agir qui remet au travail les préoccupations qui y jouaient un rôle organisateur. Pour le dire autrement, ce développement ouvre sur un pouvoir d'agir potentiel à travers lequel le sujet envisage la possibilité de «faire quelque chose » de ses préoccupations. Ce pouvoir d'agir potentiel est alors mis à l'épreuve de l'activité en classe, nécessitant pour être actualisé l'apprentissage de nouveaux usages de soi et de nouveaux gestes de métier. Les apprentissages ainsi réalisés, en permettant le recyclage des préoccupations du sujet dans des occupations renouvelées et plus efficaces pour les élèves comme pour lui, autorisent un développement de ces dernières et restaurent le pouvoir d'agir effectif du sujet. Mais les occupations initiales, celles qui ont fait l'objet de l'ACS, vont à nouveau être objets de co-analyse au cours de l'ACC, alors qu'elles se sont transformées entre-temps. À ce moment-là le sujet ne les regarde plus avec les mêmes yeux, non seulement parce que son activité est adressée à un pair, mais aussi à cause de cette transformation qui s'est opérée depuis la réalisation de l'ACS. Le développement du sens de son activité 
d'enseignement initié par cette nouvelle situation dialogique devient source potentielle d'apprentissage, dans la mesure où là encore en découvrant d'autres buts liés à des possibilités insoupçonnées dans ses occupations (Rubinstein, 2007), le sujet envisage d'autres occupations et imagine les moyens de leur réalisation, qu'il lui faudra transformer en ressources opératoires lors du retour en classe. Mais une partie des préoccupations qui se font jour s'avèrent partagées et renvoient à des dilemmes qui ne concernent pas seulement les protagonistes : l'échange entre eux laisse alors ouverte la question des voies possibles pour leur faire face. Ces préoccupations non recyclées deviennent des «post-occupations » qui sont importées dans le collectif, à qui incombe la charge de relancer le travail sur les gestes professionnels possibles pour surmonter leurs difficultés. Mais au-delà du collectif «vivant » des professionnels partenaires de l'intervention, c'est le métier lui-même, au sens d'histoire et de mémoire collective, qui est interpellé par les questions que suscitent des situations professionnelles souvent inédites et lourdes d'incertitude, que les générations antérieures n'ont pas connues, ou à tout le moins pas sous les mêmes traits, et dont la maîtrise appelle l'élaboration de nouvelles manières de faire et de penser et de nouveaux usages de soi. Il s'agit d'un enjeu dont on a essayé de montrer l'importance pour progresser vers une théorie de la formation des enseignants, mais aussi vers une conception plus pertinente de contenus et de dispositifs de formation (Durand, 2009 ; Felix, à paraitre), ce qui conduirait à envisager la formation elle-même comme une activité et à restituer l'analyse de l'activité des enseignants par rapport à cette visée de formation.

\section{Discussion}

Quelle fonction assigner à une analyse du travail dans une formation aux métiers de l'enseignement ? C'est à cette question que nous avons tenté de répondre en nous efforçant de montrer comment des matériaux issus de l'analyse de l'activité d'enseignants pouvaient se transformer en ressources de formation.

Dans la situation retenue, Guillemette et Frédérique, confrontées à l'hétérogénéité des élèves et l'obligation d'avoir à gérer des rythmes d'apprentissage différents, s'interrogent sur la manière d'initier, maintenir, réguler l'activité collective du groupe-classe tout en permettant à chacun d'apprendre du travail de tous? Entre «assurer simultanément la cohésion du groupe » et la «cohérence des apprentissages » : comment apporter à chacun des élèves une aide juste et efficace sans avoir à se diviser en 25 ? 
Le rapprochement du «cas Guillemette» à d'autres cas abordés à l'occasion d'autres interventions dans d'autres établissements scolaires et/ou de formation, ainsi que ses retouches successives résultant des reconfigurations horizontales au sein de cette collection de cas (Passeron \& Revel, 2005), nous permettent de considérer ce dernier comme un prototype clinique (Clot, 1999) mettant en évidence le développement des préoccupations des enseignants et, plus généralement, « la manière et la raison des singularités de se répéter » (Hubault, 2007, p. 84). Nous entendons par là qu'au-delà de la singularité de ce cas, il nous semble possible «[d']en extraire une argumentation de portée plus générale, dont les conclusions pourront être réutilisées pour fonder d'autres intelligibilités » (Passeron \& Revel, 2005 , p. 9) quant à l'usage de l'analyse de l'activité comme instrument de formation et à ses effets sur les processus d'apprentissage et de développement professionnels. L'enjeu n'est rien de moins que de redéfinir les contours de ressources génériques qui puissent servir de recours face à l'incertitude, par l'exploration commune de ce qui est juste ou injuste, efficace ou non. Ici, comme on l'a vu précédemment, le métier est en quelque sorte poussé dans ses retranchements par les interrogations qui ont pris progressivement corps au cours de l'intervention. Le développement de sa capacité à ré-agir face aux découvertes, aux discordances vécues et aux hypothèses «en actes » qui ont jailli dans l'analyse de l'activité dans les différents contextes (ACS et ACC, retour au collectif restreint puis élargi, retour en classe...) est alors nécessaire pour instruire, par exemple, une discussion sur les critères d'une gestion efficace et efficiente de l'hétérogénéité des élèves (commande initiale de notre intervention dans l'établissement). Mais cela ne suffit pas ; il ne suffit pas de mettre l'analyse du travail au centre du travail, encore faut-il élucider les finalités de cet acte: le développement du métier, comme répondant collectif, dépositaire d'une mémoire transpersonnelle (elle traverse les générations et chaque professionnel individuellement), constituée d'une «gamme d'actions encouragées ou inhibées dans un collectif professionnel » (Clot \& Gollac, 2014, p. 145), ouvre sur une zone potentielle d'apprentissage dont la formation peut et doit s'emparer.

Nous souscrivons ici à l'idée avancée par Clot (2007) que la formation continue, en instituant «une zone de migration fonctionnelle du savoir académique dans l'expérience et de l'expérience dans le savoir académique [peut jouer le rôle de] courroie de transmission entre la formation théorique et l'exercice du métier » (p. 91).

Toutefois, le métier n'existe pas seulement dans ses aspects transpersonnels, il est aussi impersonnel, en ce qu'il réside également dans les prescriptions et l'organisation du travail. Or les politiques éducatives et les prescriptions adressées aux enseignants tendent, depuis des 
années, à définir en dehors de ces derniers les critères de qualité de leur travail, les contraignant à utiliser massivement leur intelligence pour «gérer dans l'ombre ce que l'organisation officielle ne prend pas en charge, [alors qu'on] pourrait imaginer de l'utiliser au grand jour pour améliorer l'organisation »(Daniellou, 2010, p. 75). La rhétorique de la professionnalisation vient conforter aujourd'hui cette tendance, lorsqu'elle postule qu'il est possible de changer le travail enseignant en «exterritorialité » (Schwartz, 2000), non seulement par la prescription et l'évaluation, mais aussi par la formation. Cela conduit bien souvent cette dernière à assurer le colmatage des dysfonctionnements engendrés par des dispositifs mal conçus (Wisner, 1995), plutôt que de prêter attention au travail réel des enseignants afin de comprendre et développer avec eux la manière dont ils s'efforcent de poursuivre dans l'usage la conception forcément inachevée de ces dispositifs (Bourgeois \& Hubault, 2013).

Il importe donc que le produit du travail sur le travail, matérialisé dans l'artefact vidéo mentionné plus haut qui a permis lors des retours au collectif d'instrumenter la réflexion sur les possibilités de relance de l'activité, se transforme en un autre instrument, enrichi des avancées au sein du collectif élargi mais aussi des questionnements qui y sont demeurés ouverts au service cette fois d'un dialogue avec la direction de l'établissement à l'origine de la demande et, plus largement, l'institution, notamment les services décentralisés de l'Éducation nationale en charge de la formation et de l'innovation, visant le développement impersonnel du métier dans l'organisation. De ce point de vue, on peut dire de ce type d'analyse du travail qu'elle est une formation en tant que telle: «Mais c'est alors une formation elle-même transformée en travail » (Clot, 2000, p. 155).

Pour autant, il ne s'agit pas ici d'introduire un nouvel impérialisme dans le champ de l'éducation et de la formation en imposant une « entrée activité » comme solution à tous les maux de la formation professionnelle des adultes.

En revanche, comme nous avons tenté de le montrer à travers le «cas Guillemette », nous défendons la pertinence qu'il y a à promouvoir, en formation, le passage d'une posture où les outils sont pensés uniquement par rapport à l'activité des élèves à une posture où l'on conçoit des outils pour faciliter l'activité de l'enseignant. De ce point de vue, lorsque le temps de la recherche succède à celui de l'intervention, les matériaux issus de la co-analyse, qui ont servi de ressources au service de visées transformatives et formatives, peuvent alors changer de statut en devenant des ressources au service de visées épistémiques. On retiendra entre autres ici ce que l'analyse de ces matériaux permet de révéler quant à la tâche scolaire comme instrument du travail enseignant. En effet celle-ci y apparait dotée d'une «multi- 
fonctionnalité » (Laurent \& Saujat, sous presse) : ergonomique d'abord, comme moyen de mettre les élèves au travail et de générer un confort professionnel relatif ; «doublement didactique » ensuite, au sens où la tâche a non seulement le statut d'objet de conception voué à devenir un moyen d'apprentissage pour les élèves, mais aussi celui de moyen de l'activité de l'enseignant qui apprend de ses élèves grâce à l'activité que la tâche déclenche, tout en étant simultanément lui-même un moyen de l'activité de ces derniers.

L'intervention, en mobilisant l'analyse du travail comme instrument de formation, vise à initier un triple développement: a) des situations examinées, par la remise en travail dont elles font l'objet, b) des sujets engagés, par la découverte des dimensions non réalisées ou non perçues de leur activité et la conception des façons de s'en affranchir en leur offrant un destin possible, et c) du collectif, par la reconnaissance ou le dépassement des modalités communes de l'action. Les traces «développementales» ainsi produites constituent alors « un support irremplaçable à la production de savoirs »(Daniellou, 2007, p. 7), tout particulièrement sur les organisateurs de l'activité enseignante et de son développement.

Ce parti pris ouvre vers des collaborations évidentes avec d'autres disciplines des sciences de l'éducation et du travail, notamment avec la didactique professionnelle et les didactiques des disciplines, en vue de redessiner les contours des sciences de la formation. 


\section{Références bibliographiques}

Bakhtine, M. (1970). La poétique de Dostö̈evski. Paris : Seuil.

Bakhtine, M. (1977). Le marxisme et la philosophie du langage. Paris : Éditions de Minuit.

Bakhtine, M. (1984). Esthétique de la création verbale. Paris : Gallimard.

Bourgeois, E., \& Durand, M. (Eds). (2012). Apprendre au travail. Paris : Puf.

Bournel Bosson, M. (2011). Rapports développementaux entre langage et activité. In B. Maggi (Ed.), Interpréter l'agir : un défi théorique (pp. 147-162). Paris : Puf.

Bronckart, J.-P. (2001). S'entendre pour agir et agir pour s'entendre. In J.-M. Baudouin \& J. Friedrich (Eds), Théories de l'action et éducation (pp. 133-154). Bruxelles : De Boeck.

Canguilhem, G. (1930, novembre). De l'introspection. Libres propos.

Clot, Y. (2000). La formation par l'analyse du travail : pour une troisième voie. In B. Maggi (Ed.), Manières de penser, manières d'agir en éducation et formation (pp. 133-156). Paris : Puf.

Clot, Y. (2007). De l'analyse des pratiques au développement des métiers. Éducation et didactique, 1, 83-94.

Clot, Y., \& Gollac, M. (2014). Le travail peut-il devenir supportable ? Paris : Armand Colin.

Clot, Y., \& Leplat, J. (2005). La méthode clinique en ergonomie et en psychologie du travail. Le travail humain, 68, 289-316.

Daniellou, F. (1995). La construction sociale de et par l'analyse du travail. Performances Humaines \& Techniques, hors-série séminaire Paris 1, 25-29.

Daniellou, F. (1996). L'ergonomie en quête de ses principes. Toulouse : Octarès.

Daniellou, F. (2007). Manifeste. Éducation Permanente, 170, 7-11.

Daniellou, F. (2010). Les mondes du travail. In L. Terry (Ed.), Le travail intenable. Résister collectivement à l'intensification du travail (pp. 29-94) Paris : La Découverte.

Durand, M. (2009). La conception d'environnements de formation sous le postulat de l'enaction. In M. Durand \& L. Filliettaz (2009), Travail et formation des adultes (pp. 191215). Paris : Puf.

Durand, M., \& Filliettaz, L. (Eds). (2009). Travail et formation des adultes. Paris : Puf. Faïta, D. (2007). L’image animée comme artefact dans le cadre méthodologique d'une analyse clinique de l'activité. Activités, 4(2), 3-15. Récupéré de http://www.activites.org/v4n2/v4n2.pdf 
Faïta, D., \& Vieira, M. (2003). Réflexions méthodologiques sur l'autoconfrontation croisée. Skholê, numéro spécial « Métier enseignant, organisation du travail et analyse de l'activité », $57-68$.

Felix, C. (2014). Du travail des «collectifs» à de nouvelles modalités de formation professionnelle : l'histoire du GAP. Questions vives, Recherches en éducation, 6, 21.

Felix, C. (à paraitre). De l'intervention-recherche à la formation professionnelle des enseignants : quelle didactisation du travail réel des enseignants ? Recherche et Formation, 75 .

Felix, C., \& Saujat, F. (2007, août). Les débuts dans le métier enseignant. Congrès International AREF, Strasbourg.

François, F. (1993). Pratiques de l'oral. Paris : Nathan.

François, F. (1996). Communication, interaction, dialogue... Remarques et questions. Le Français aujourd'hui, 113, 11-23.

Hubault, F. (2007). Nature d'intervention, nature de savoir. Éducation Permanente, 170, $77-$ 85.

Jobert, G. (2000). Dire, penser, faire. À propos de trois métaphores agissantes en formation d'adultes. Éducation Permanente, 143, 7-28.

Laurent, Y., \& Saujat, F. (sous presse). L'intervention en milieu de travail éducatif entre développement de l'activité professionnelle et développement de l'activité de connaissance. Carrefours de l'Éducation.

Leblanc, S., Ria, L., Dieumegard, G., Serres G., \& Durand M. (2008). Concevoir des dispositifs de formation professionnelle des enseignants à partir de l'analyse de l'activité dans une approche enactive. @ctivités, 5(1), 58-78.

Mayen, P. (1999). Des situations potentielles de développement. Éducation Permanente, 139, 65-86.

Passeron, J.-C., \& Revel, J. (Eds). (2005). Penser par cas. Paris : Éditions de l'EHESS.

Rubinstein, S.L. (2007). Nouvelles figures de l'activité humaine. Anthologie de textes choisis et édités par V. Nosulenko et P. Rabardel. Toulouse : Octarès \& Éditions Maison des sciences de l'homme.

Saujat, F. (2007). Enseigner: un travail. In V. Dupriez \& G. Chapelle (Eds), Enseigner (pp. 179-188). Paris : Puf.

Saujat, F., \& Serres, G. (2015, janvier). L'activité de l'enseignant d'EPS entre préoccupations et « occupations » : un point de vue développemental. eJRIEPS. 
Schwartz, Y. (2000). Le paradigme ergologique ou un métier de philosophe. Toulouse: Octarès.

Wallon, H. (1969). Les milieux, les groupes et la psychogenèse de l'enfant. Enfance, numéro spécial, 287-296. (Original publié en 1954).

Wisner, A. (1995). Réflexions sur l'ergonomie. Toulouse : Octarès. 